\title{
Neurofilament light chain in demyelinating conditions of the central nervous system: a promising biomarker
}

\author{
Silvia Bozzetti, Sergio Ferrari, Alberto Gajofatto, Sara Mariotto \\ Neurology Unit, Department of Neuroscience, Biomedicine and Movement Sciences, University of Verona, Verona 37134, Italy. \\ Correspondence to: Dr. Sara Mariotto, Neurology Unit, Department of Neuroscience, Biomedicine and Movement Sciences, \\ University of Verona, Policlinico GB Rossi, P.le LA Scuro 10, Verona 37134, Italy. E-mail: sara.mariotto@gmail.com
}

How to cite this article: Bozzetti S, Ferrari S, Gajofatto A, Mariotto S. Neurofilament light chain in demyelinating conditions of the central nervous system: a promising biomarker. Neuroimmuno/ Neuroinflammation 2021;8:1-13.

http://dx.doi.org/10.20517/2347-8659.2020.26

Received: 27 Mar 2020 First Decision: 17 Jun 2020 Revised: 23 Jun 2020

Accepted: 16 Jul 2020 Fisrt online: 15 Aug 2020 Published: 21 Mar 2021

Academic Editor: Roberta Magliozzi Copy Editor: Cai-Hong Wang Production Editor: Tian Zhang

\begin{abstract}
Neurofilaments are the major structural proteins of the neuronal cytoskeleton and are classified according to molecular weight into heavy, intermediate, and light chains. They are released into the interstitial fluid and cerebrospinal fluid (CSF) as a consequence of axonal damage. In particular, the light chain (NfL) represents the most abundant and soluble subunit and has been demonstrated to be increased in the CSF of patients with inflammatory, degenerative, vascular, or traumatic injuries in correlation with clinical and radiological activity. Similar results have been obtained measuring serum NfL with high-sensitivity single-molecule array, which enables reliable and repeatable measurement of the low NfL concentrations in serum. In particular, CSF and serum $\mathrm{NfL}$ values are strongly correlated in patients with multiple sclerosis (MS) and have been demonstrated to be increased in patients with MS and clinically isolated syndromes (CIS) in accordance with clinical and radiological activity. NfL levels increase in patients with a recent relapse and seem to predict cognitive impairment, longterm outcome, and conversion of CIS to MS. The few available data on patients with other demyelinating diseases suggest that $\mathrm{NfL}$ levels are also increased in neuromyelitis optica spectrum disorders and related conditions in correlation with attack severity, suggesting that axonal damage may occur in these disorders. We herein report and discuss published data on the role of $\mathrm{NfL}$ as a possible predictor of disease activity, clinical outcome and treatment response in patients with demyelinating conditions of the central nervous system.
\end{abstract}

Keywords: Neurofilament light chain, multiple sclerosis, clinically isolated syndromes, radiologically isolated syndrome, neuromyelitis optica spectrum disorders, myelin oligodendrocyte glycoprotein, aquaporin-4



(C) The Author(s) 2021. Open Access This article is licensed under a Creative Commons Attribution 4.0 International License (https://creativecommons.org/licenses/by/4.0/), which permits unrestricted use, sharing, adaptation, distribution and reproduction in any medium or format, for any purpose, even commercially, as long as you give appropriate credit to the original author(s) and the source, provide a link to the Creative Commons license, and indicate if changes were made.

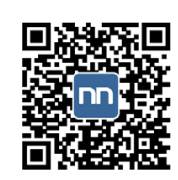




\section{INTRODUCTION}

Inflammatory demyelinating diseases (IDD) represent a spectrum of heterogeneous disorders affecting the central nervous system (CNS). Multiple sclerosis (MS) is classified as a chronic, immune-mediated, demyelinating disorder, and it is the most well-known disease of this group ${ }^{[1]}$. Neuromyelitis optica spectrum disorders (NMOSD), which preferentially involve the spinal cord and optic nerve ${ }^{[2]}$, and acute disseminated encephalomyelitis (ADEM), a typically monophasic disease of children ${ }^{[3]}$, are also part of CNS IDD. Other acute inflammatory conditions including idiopathic optic neuritis ${ }^{[4]}$ and acute transverse myelitis ${ }^{[5]}$ also enter in the differential diagnosis. A major discovery in this field was the association between NMOSD and serum aquaporin $4 \operatorname{IgG}(\mathrm{AQP} 4-\mathrm{IgG})$, confirming that it is a different disease from MS and needs different treatment ${ }^{[6]}$. On the other hand, the association of serum anti-myelin oligodendrocyte glycoprotein (MOG)-Abs with ADEM, NMOSD and other demyelinating events also clarify the final diagnosis in many conditions previously classified as "idiopathic" ${ }^{\text {" }}{ }^{[]}$. In addition to the difficulty in the diagnostic process, one of the main issues of IDD is the correct assessment of disease activity and the prediction of long-term prognosis. Different clinical scales, radiological parameters, and biological markers have been studied, with the aim of identifying reliable and easily accessible measures of disease activity, treatment response, and prognosis in these conditions. Neurofilament proteins recently emerged as a promising biomarker in this context. Neurofilaments are cylindrical proteins located in dendrites, soma and, in particular, axons of neurons, with their specific role in conferring structural stability and promoting axonal growth and intracellular transport. They are classified as intermediate filaments (i.e., $10 \mathrm{~nm}$ in diameter, intermediate between actin and myosin) and include neurofilament light chain (NfL), neurofilament middle chain, neurofilament heavy chain $(\mathrm{NfH})$, and $\alpha$-internexin, depending on the length of the carboxyterminal region. Since NfLs are the most abundant and soluble subunit among intermediate filaments, research has mainly focused on them ${ }^{[8]}$. Low levels of NfL are constantly released from axons under normal conditions, in an age-dependent manner. However, as a consequence of axonal damage due to inflammatory, degenerative, vascular, or traumatic injury, NfL release significantly increases. After reaching the interstitial fluid, NfL are detectable in CSF and in serum at lower but comparable levels ${ }^{[9]}$. Enzymelinked immunosorbent assay (ELISA) technology allows the measurement of the higher NfL values present in CSF; however, it is not sensitive enough to measure the significantly lower serum/plasma levels. The recent development of ultrasensitive electrochemiluminescence-based immunoassay [in particular, singlemolecule immunoassay, single-molecule array (SiMoA) technology] enables reliable measurement of the low NfL concentrations in serum and the monitoring of minor changes over time ${ }^{[10]}$. This single-molecule immunoassay is based on antibody capture agents bound to the surface of paramagnetic microbeads containing approximately 250,000 attachment sites. The beads are added to the sample solution and then incubated with a second biotinylated detection antibody and beta-galactosidase-labeled streptavidin. In this manner, each bead that has captured a single protein molecule is labeled with an immunocomplex. During the detection process, a fluorescent signal is generated in sealed wells that contain beads combined with immuno-captured and enzyme-labeled protein molecules. Concentrations are determined digitally to further increase the sensitivity of the assay. With this recently developed technique, a significant increase in serum NfL levels has been demonstrated in different conditions, including Alzheimer's disease ${ }^{[1]}$, Creutzfeldt-Jakob disease ${ }^{[12]}$, frontotemporal dementia ${ }^{[13]}$, amyotrophic lateral sclerosis ${ }^{[14]}$, parkinsonian disorders $^{[15]}$, traumatic brain injury ${ }^{[16]}$, stroke ${ }^{[17]}$, peripheral neuropathies ${ }^{[18]}$, autoimmune encephalitis ${ }^{[19]}$, and in particular $\mathrm{MS}^{[20]}$, in correlation with disease activity and post-mortem neurodegeneration ${ }^{[21]}$. The lower invasiveness of serum NfL measurement allows repeatable analyses over time and expands the potential utility of NfL as a biomarker of disease activity and treatment response in a wide spectrum of neurological disorders. Although the actual applicability of this assay in daily clinical practice is still limited and influenced by critical aspects that need to be considered for the correct interpretation of this measurement ${ }^{[2]}$, the development of novel ultrasensitive assays and the extensive applicability of serum NfL measurement have provided major advantages in this field. 


\section{NFL IN SUBJECTS WITH CLINICALLY ISOLATED SYNDROME AND RADIOLOGICALLY ISOLATED SYNDROME}

Clinically isolated syndrome (CIS) is defined as the first episode of clinical symptoms that potentially precedes MS. About $85 \%$ of patients with CIS experience a second clinical episode, thus evolving into MS within the subsequent 10 years ${ }^{[23]}$. Radiologically isolated syndrome (RIS) is defined by MRI findings suggestive of MS in the absence of clinical symptoms. Progression to MS usually occurs in approximately $66 \%$ of patients with RIS ${ }^{[24]}$. The uncertain evolution and variable long-term prognosis of patients with CIS/ RIS make attractive the discovery and validation of specific biomarkers able to identify cases that will have future clinical attacks.

In this scenario, the potential utility of CSF NfL as a predictive marker of disease evolution has been recently explored. In particular, Håkansson et al ${ }^{[25]}$ analyzed CSF levels of NfL, NfH, and other neurodegenerative and inflammatory markers in 19 patients with CIS, 22 cases with relapsing remitting multiple sclerosis (RRMS), and 22 sex- and age-matched healthy controls in a prospective longitudinal study. Disease activity (i.e., radiological lesion load, presence of relapses, and disability worsening) was recorded at 2 years follow-up and compared with the levels of specific biomarkers measured at baseline. Interestingly, NfL values were the only prognostic marker potentially able to predict disease activity in subjects with CIS and MS. A different study compared CSF NfL levels with brain volume measured in 41 patients with CIS and 30 controls and demonstrated that NfL values were higher in subjects with CIS and were inversely associated with grey matter volume $e^{[26]}$. CSF NfL and progranulin levels were also evaluated in subjects with RIS and compared with those determined in subjects with CIS, MS, and healthy controls $\mathrm{s}^{[27]}$. Interestingly, NfL levels were significantly lower in subjects with CIS and RIS in comparison with patients with RRMS and primary progressive multiple sclerosis (PPMS), suggesting that the detection of this biomarker could parallel clinical evolution in these disorders. In addition, CSF NfL values recently emerged as an independent risk factor for clinical conversion in subjects with RIS with higher levels associated with shorter time to progression ${ }^{[28]}$.

After the discovery of highly sensitive techniques able to measure the lower values of NfL in plasma/serum, the potential utility of serum NfL in predicting CIS conversion to MS has been explored. Disanto et al. ${ }^{[29]}$ demonstrated that serum NfL levels are higher in subjects with CIS in comparison with healthy controls and are associated with T2 hyperintense MRI lesions, gadolinium-enhancing lesions, and disability score at CIS diagnosis, but do not allow subjects with CIS who will convert to definite MS after a short interval ( $n=100)$ to be distinguished from subjects with CIS who will not evolve $(n=100)$. The potential effect of riluzole treatment in subjects with CIS and early MS ( $n=22$ CIS/MS cases randomized to riluzole and $n=21$ to placebo) in comparison with clinical parameters and serum NfL/NfH values was also analyzed. Despite the absence of treatment effect, the authors demonstrated that NfL levels correlated with Expanded Disability Status Scale (EDSS) changes and neuropsychological outcome ${ }^{[30]}$. Furthermore, higher NfL levels at baseline were associated with a more rapid decrease in brain volume and predicted higher number of enhancing lesions, confirming the role of NfL as a potential marker of neuronal and axonal damage in CIS and early MS. Finally, in a case-control study performed among US military that analyzed serum samples of 60 subjects asymptomatic at time of sampling who then developed MS (6 years later as a median), the authors observed increased serum NfL levels in cases that would develop MS in comparison to healthy controls, demonstrating a potentially useful value of serum NfL in predicting future development of/ evolution to $\mathrm{MS}^{[31]}$.

Taken together, available data support the role of CSF NfL levels as a predictive and prognostic marker of CIS/RIS. On the other hand, serum NfL levels are increased in patients with a recent relapse and a high number of T2/enhancing lesions on MRI. Despite the more evident association between NfL levels and 
disability at baseline than at follow-up, recent studies indicate that serum NfL values could also be useful to predict MS conversion in patients with a first demyelinating event, with lower NfL levels indicating reduced risk of receiving a future MS diagnosis ${ }^{[32]}$.

\section{NfL IN RELAPSING-REMITTING MS}

RRMS is the most common form of MS, involving $85 \%$ of affected patients ${ }^{[33]}$. RRMS is characterized by discrete and clearly definite attacks lasting days to weeks, followed by periods of partial/complete remission in the absence of progressive clinical deterioration ${ }^{[33]}$. Disease severity and lifelong prognosis of patients with RRMS are highly variable, so that a correct subclassification of this condition according to risk of future disease activity and final disability is of utmost importance to guide prompt therapeutic strategies ${ }^{[34]}$. The combination of clinical and radiological indicators of disease activity have long been used, despite the high costs and incomplete predictive strength. To overcome these limitations and increase the sensitivity of outcome prediction, the potential utility of different serum and CSF biomarkers have been explored over the last years using high-sensitivity technology ${ }^{[20,25,35,36]}$. In particular, NfL levels have been analyzed and compared to glial fibrillary acidic protein $(\mathrm{GFAP})^{[25,36]}, \mathrm{S} 100 \mathrm{~B}$, neuron-specific enolase (NSE) $)^{[5]}$, chitinase 3-like 1 (CHI3L1) levels ${ }^{[2,25]}$, and to a panel of chemokines, matrix metalloproteinase-9, and ostepontin ${ }^{[25]}$ with divergent data on the value of combining these biomarkers. The comparison between these biomarkers and previously recognized clinical/radiological parameters of disability aimed to distinguish MS patients and healthy controls, to improve the prediction of ongoing and future disease activity, to better predict long-term outcome in terms of brain and spinal cord atrophy, final disability and risk of progression, and to evaluate response to disease modifying therapy $(\mathrm{DMT})^{[35]}$.

The potential role of NfL in MS was proposed for the first time by Lycke et al ${ }^{[37]}$ in 1998 , who detected increased levels of NfL in the CSF of RRMS patients in comparison with healthy controls. The authors also demonstrated a significant correlation between NfL values and disability as assessed by EDSS score, exacerbation rate, and time from last relapse. These findings gave important insight into MS pathogenetic mechanisms, suggesting the presence of axonal damage in subjects with a relapsing-remitting course and postulating a contribution of axonal pathology to disability ${ }^{[37]}$. Subsequently, the potential utility of measuring NfL levels in subjects with RRMS, monitoring longitudinal levels over time, offered indirect cues to the understanding of NfL kinetics in blood and $\operatorname{CSF}^{[2,35,38-40]}$. Different studies displayed substantial differences in terms of the matrix analyzed (serum, plasma, or CSF) and the performance of the assays used, giving a clear spectrum of the evolution of the detection techniques and their related sensitivity ${ }^{[41]}$. These assays ranged from a second-generation ELISA ${ }^{[37,39,42-44]}$, to a third-generation electrochemiluminescence technology ${ }^{[9,45]}$ and, finally, to a fourth-generation $\operatorname{SiMoA}^{[9,20,25,38,40,46-48]}$ that enables a reliable and highly sensitive quantification and monitoring of serum/plasma NfL levels. In particular, the SiMoA novel ultrasensitive technology increases the sensitivity of the assay allowing comparisons between pathological and normal NfL values using small sample volume ${ }^{[22]}$. This technical improvement, together with the demonstration of a clear correlation between serum/plasma and CSF NfL levels ${ }^{[45,49]}$, now enables the reliable measurement of $\mathrm{NfL}$ in blood samples, avoiding more costly and invasive procedures such as lumbar puncture. This concept further supports the potential use of NfL as a promising biomarker useful for longitudinal monitoring of disease activity and treatment response.

\section{$\mathrm{NfL}$ values help to distinguish patients with RRMS from healthy controls}

The first objective when investigating NfL levels in RRMS patients was to evaluate whether this biomarker could be useful to differentiate patients from healthy controls. Significantly higher NfL levels in both $\operatorname{CSF}^{[9,25,37,39,46,48]}$ and serum ${ }^{[9,38,45,48]}$ have been reported in patients $v s$. healthy controls using different techniques. However, the substantial variation in NfL values observed in different studies prevented the identification of a reproducible cut-off and suggested a great inter-individual variability, possibly influenced by differences in measurement sensitivity, but not clearly related to demographic characteristics, sample 
storage, or disease duration ${ }^{[35]}$. Actually, an attempt to identify specific diagnostic cut-offs for CSF, plasma, and serum through a receiver operating characteristic analysis ${ }^{[46]}$ was reported in a recent prospective phase IV study conducted with the aim of evaluating the effect of dimethyl-fumarate on NfL values. The authors identified specific NfL cut-offs to discriminate between MS patients and healthy controls with a $100 \%$ specificity, i.e., $807.5 \mathrm{pg} / \mathrm{mL}$ ( $80 \%$ sensitivity) for CSF, $13.0 \mathrm{pg} / \mathrm{mL}$ ( $47 \%$ sensitivity) for plasma, and $15.6 \mathrm{pg} / \mathrm{mL}$ (43.2\% sensitivity) for serum ${ }^{[46]}$.

\section{NfL levels correlate with disease activity at sampling}

NfL levels have also been demonstrated to correlate with disease activity in RRMS patients, which is commonly assessed using a combination of different surrogate biomarkers, including clinical parameters such as relapse-rate, and MRI signs (i.e., the presence of gadolinium-enhancing lesions or new or unequivocally enlarging T2 hyperintense lesions $)^{[34]}$. In particular, in one of the first attempts to correlate NfL levels with disease activity, the authors demonstrated that CSF NfL values were significantly increased 2-3 months after a clinical relapse and tended to gradually decrease thereafter ${ }^{[37]}$. This pioneer finding was confirmed using more sensitive fourth-generation methods, which allowed the demonstration of increased $\mathrm{CSF} /$ serum NfL concentrations in patients who experienced a relapse within 3 months before sample collection, compared to those in remission ${ }^{[49]}$. In addition, a robust association between NfL values and radiological parameters of disease activity has been demonstrated. In particular, CSF/serum NfL levels are significantly higher in patients with gadolinium-enhancing lesions ${ }^{[9,20,49]}$ and with new or enlarging $\mathrm{T} 2$ lesions ${ }^{[20,40,50]}$. Moreover, NfL concentration progressively increases in correlation with the number of contrast enhanced lesions ${ }^{[9,38,50]}$ and $\mathrm{T} 2$ lesion load ${ }^{[38]}$ detected in both brain and spinal cord ${ }^{[9]}$. As for correlations between NfL values and clinical measure of disability, a robust correlation has been reported between NfL levels and EDSS score at sampling ${ }^{[9,35,38,49]}$. In a recent cross-sectional study performed on two Swiss MS cohorts, serum NfL concentration at baseline emerged as an indicator of previous clinical disease activity, being significantly associated with a relapse within 60 days before sampling, mean annual relapse rate in the last 1 and 2 years, and with the probability of EDSS worsening during the last 6 and 12 months ${ }^{[9]}$.

\section{$\mathrm{NfL}$ levels have a role in the prediction of future disease activity}

Attention has more recently been devoted to the possible prognostic role of NfL, to determine whether its concentration could correlate with clinical and radiological biomarkers of future disease activity ${ }^{[9,40,45,47-49,51,52]}$, with treatment respons $\mathrm{e}^{[20,38,39,42,46,49,53]}$, and with progression to a secondary progressive course ${ }^{[54]}$.

In particular, serum NfL levels at baseline have displayed a significant association with the number of clinical relapses in the subsequent 18 months $^{[40]}$ and consequently with an increase in annual relapse rate at 1 and 2 years follow-up ${ }^{[9]}$, supporting the value of this biomarker in predicting future disability. A strong and independent correlation between serum NfL levels above the 90th percentile of healthy controls values and EDSS worsening in the following 12 months was recently observed in a cohort including 189 patients with RRMS, 70 progressive cases, and 259 healthy controls ${ }^{[51]}$. Different studies have also confirmed a significant association between high serum NfL values at baseline and radiological hallmarks of disease activity/progression during the follow-up, i.e., new T2-lesions and brain volume loss during the subsequent 4 years $^{[48]}$ and brain/spinal cord volume loss as measured after 2 and 5 years from blood sampling ${ }^{[51]}$. In particular, Barro et al.$^{[51]}$ reported a correlation between the percentage of brain/spinal cord volume changes and serum NfL levels, that in a multivariate model remained the only predictors of brain volume loss at 2 years follow-up.

\section{$\mathrm{NfL}$ levels help to predict long-term outcome}

The possible role of NfL in predicting long-term clinical and radiological outcome in RRMS patients has been investigated assessing serum and/or CSF NfL values in the course of a phase 3 randomized placebocontrolled trial of intramuscular interferon-beta ${ }^{[47]}$. A robust association emerged between CSF NfL 
concentration measured at year 2 from the beginning of the trial and EDSS changes, as well as brain atrophy, expressed by brain parenchymal fraction (BPF) change at 8 years follow-up. Similarly, serum NfL levels at 3 years displayed a correlation with both BPF and EDSS changes at 8 years follow-up, whereas $\mathrm{NfL}$ values at 4 years showed a significant association with EDSS changes over 15 years $^{[47]}$. CSF NfL concentrations at year 2 and serum NfL levels at year 3 in the upper tertile predicted an increased risk of reaching an EDSS score of 6.0 or higher at 8 years follow-up ${ }^{[47]}$. During the last years, a composite clinical and paraclinical definition of "no disease activity" (NEDA) that includes the absence of relapses, disability worsening, and new or enlarging MRI lesions was proposed as the main target of MS treatment ${ }^{[55]}$. Several studies have reported that NfL levels at baseline are significantly lower in patients with no evidence of activity during the subsequent follow-up ${ }^{[25,48]}$, therefore showing an accuracy of $85 \%$ in correctly classifying NEDA3 cases over the following 2 years ${ }^{[25]}$. These findings have led to the proposal of expanding the concept of NEDA, taking into consideration also the assessment of brain atrophy and the evaluation of serum and CSF biomarkers, including $\mathrm{NfL}^{[56]}$. Finally, a recent longitudinal study in a Norwegian cohort of 44 patients with newly diagnosed MS and a long-term follow-up of 10 years demonstrated that CSF NfL values were significantly higher in patients evolving from RRMS to secondary progressive multiple sclerosis (SPMS) over 5 years, suggesting a possible role of NfL in predicting the risk of a secondary progressive disease course ${ }^{[54]}$.

\section{$\mathrm{NfL}$ levels as a measure of treatment response}

Besides the role as a diagnostic and disease activity biomarker, one of the most attractive applications of $\mathrm{NfL}$ is their possible use in monitoring therapeutic response. The evidence that serum NfL levels are lower in patients under $\mathrm{DMT}^{[9,49]}$ and that initiation and escalation of such therapies significantly decrease NfL concentrations $^{[49]}$ has further confirmed this hypothesis. In particular, starting on an IFNB-1a therapy led to a sustained reduction of serum NfL levels over the following 12 and 24 months $^{[20]}$. Natalizumab initiation resulted in a 3 -fold reduction in CSF NfL values, which reached levels compatible with those measured in healthy controls ${ }^{[39]}$. The efficacy of fingolimod in reducing NfL concentration in serum ${ }^{[38]}$ and $\mathrm{CSF}^{[42]}$ has been demonstrated, also in comparison with $\mathrm{IFN}^{[38]}$, in a phase 3 placebo-controlled clinical trial (FREEDOMS) ${ }^{[38,42]}$ and in a phase 3 active-controlled $v s$. IFN trial (TRANSFORMS) ${ }^{[38]}$. NfL levels have been reported to be significantly reduced by $73 \%$ in CSF, $69 \%$ in serum, and $55 \%$ in plasma 1 year after dimethyl fumarate initiation in a prospective open-label phase 4 clinical trial designed to evaluate the effect of dimethyl fumarate in a cohort of newly-diagnosed RRMS patients (TREMEND). NfL values were similar to those measured in healthy controls in all serum samples, in $96 \%$ of plasma samples, and in $72 \%$ of CSF samples of treated patients 1 year after treatment initiation ${ }^{[46]}$. Finally, the therapeutic switch from IFNB or glatiramer acetate to rituximab has been demonstrated to produce a significant (i.e., $21 \%$ ) reduction of CSF NfL values during the subsequent year in a cohort of 75 patients with $\mathrm{RRMS}^{[53]}$. The role of NfL as drug-response markers has recently been confirmed in a study analyzing the distribution of NfL in RRMS patients starting DMTs and the evolution of NfL values over time. The authors observed that the reduction in plasma NfL concentrations under DMT differed according to specific drugs, although levels were also influenced by baseline characteristics, clinical improvement, and possibly NfL kinetics. In particular, the largest reduction in NfL values was noted on treatment with alemtuzumab and the lowest on teriflunomide, while reduced NfL levels similar to that observed under treatment with alemtuzumab were noted under dimethyl fumarate, fingolimod, and natalizumab. However, groups were not homogeneous for characteristics influencing NfL levels, including age, disease duration, and disease severity, potentially resulting in an indication bias, which the authors tried to overcome with statistical adjustments for baseline characteristics $^{[57]}$.

Taken together, these data led to serum NfL being proposed as a candidate and useful biomarker for surveilling subclinical activity in clinically stable RRMS patients ${ }^{[49]}$ and for measuring and predicting disease activity and treatment response, although commonly accepted cut-off values are still lacking and NfL concentrations are not comparable between different studies. 


\section{NFL IN PROGRESSIVE MS}

PPMS is characterized by progressive neurological decline from disease onset, without experiencing attacks, and accounts for $15 \%$ of MS cases at presentation. SPMS in characterized by progression occurring after a $\mathrm{RR}$ course and involves about $50 \%$ of cases after 15 years $^{[1]}$. In this context, the potential role of NfL levels in predicting and quantifying disease progression has been explored. In one of the first studies considering 95 patients with MS and a long-term follow-up (median 14 years, range 8-20), high CSF NfL levels were associated with an unfavorable prognosis and with conversion to SPMS ${ }^{[58]}$. Although divergent CSF NfL values have been observed in patients with PPMS and SPMS, the authors suggested that NfL is a useful prognostic biomarker under these conditions ${ }^{[59,60]}$. However, the absence of correlation between NfL levels and disease duration or disease severity, measured with EDSS, led to the idea that CSF NfL levels could not properly reflect disease severity in PPMS ${ }^{[27]}$. The slow axonal degeneration occurring in subjects with PPMS together with the more robust NfL increase in the course of acute axonal damage could explain these results. Different studies further support this hypothesis. Damasceno et al. ${ }^{[61]}$ analysed CSF NfL values in consecutive patients with MS, including 32 subjects with RRMS and 15 with progressive MS, and correlated $\mathrm{NfL}$ values with radiological and clinical variables. Interestingly, NfL levels were significantly increased in patients with RRMS in association with cortical lesions and relapses, whereas they were not different in patients with progressive MS in comparison with healthy controls. Sellebjerg et al. ${ }^{[62]}$ measured CSF NfL levels in 26 patients with PPMS, 26 with SPMS, and 24 healthy controls and observed higher values in cases with active progressive MS in comparison with those with inactive progressive MS, thus supporting the role of NfL in distinguishing active $v s$. inactive cases. These data further confirm the specific association between NfL concentration and active disease at sampling, which has a significant impact on axonal damage ${ }^{[6]]}$. Partially divergent data emerged according to a recent meta-analysis of case-control studies, where three times higher CSF NfL levels were observed in 158 patients with progressive MS in comparison with healthy controls, although significantly lower values were detected in progressive $v s$. relapsing cases. NfL values tended to be higher, although not significantly different, in RRMS on remission (229 patients) in comparison with patients with progressive MS $(158)^{[63]}$. In addition to the lower levels measured in cases with progressive vs. relapsing MS, a correlation between CSF levels of sCD27 (a soluble marker of T-cells) and NfL values in subjects in progression before and after treatment with natalizumab (17 patients) and methylprednisolone ( 23 patients) was reported, suggesting a connection between residual inflammation and axonal damage and a role of these biomarkers in monitoring treatment response ${ }^{[64]}$.

The analysis of serum NfL values in patients with progressive MS further confirmed previous observations on CSF NfL measurement. In particular, higher values of serum NfL in the presence of disease activity, defined as a clinical relapse or new gadolinium-enhancing lesions on MRI, were reported in a cohort of 286 patients with MS, including both RRMS and progressive cases (19 PPMS and 63 SPMS) ${ }^{[49]}$. In a longitudinal study, Disanto et al. ${ }^{[9]}$ examined paired serum and CSF samples of different subjects (CIS $n=48$, RIS $n=$ 13, RRMS $n=62$, PPMS $n=16$, and SPMS $n=3$ ) and confirmed the strong association between CSF and serum NfL levels and the presence of 42 -fold lower values in serum. A more striking association between NfL values and disability, measured with EDSS, was noted in cases with CIS/RRMS than in those with PPMS/SPMS, once again reflecting the predominant axonal damage occurring in active cases.

A study including subjects with CIS, RRMS, PPMS, and SPMS detected an association between the probability of EDSS worsening and the increase in serum NfL values, with serum NfL levels reflecting future disease progression in terms of brain and cervical spinal cord atrophy. The authors also confirmed the association between serum NfL levels and spinal cord volume loss in patients with PPMS, even in the absence of radiological signs of inflammation, thus supporting the correlation between axonal damage and spinal cord atrophy in the course of disease progression ${ }^{[51]}$. More recently, Ferraro et al ${ }^{[65]}$ specifically studied 27 patients with PPMS and 43 with SPMS (mean follow-up of 25 months) and demonstrated a positive correlation between plasma NfL values and disability assessed with EDSS, together with an 
increase in NfL levels over time on repeated measurements. Although data on the difference of NfL levels between patients with a progressive $v s$. relapsing course are divergent, a recent systematic review confirmed that among subjects with a progressive course, higher levels are observed in those with increased clinical and radiological evidence of disease activity. The impact of disability and the possible role of NfL in predicting future disability is still debated. Finally, treatments with DMTs including natalizumab, rituximab, fingolimod, ocrelizumab, and mitoxantrone seem to affect plasma NfL levels. Unestablished treatments, first-line DMTs, or neuroprotective treatments seem less effective in influencing NfL values ${ }^{[66]}$.

\section{NFL IN NMOSD AND RELATED DISORDERS}

NMOSD is an inflammatory CNS syndrome currently diagnosed on the basis of clinical, neuroimaging and laboratory features ${ }^{[2]}$. The most typical presentations of NMOSD include acute (usually bilateral) optic neuritis with severe visual acuity impairment and longitudinally extensive transverse myelitis (LETM), typically presenting with severe symptoms including paraplegia, bowel/bladder dysfunctions, and sensory $\operatorname{loss}^{[67,68]}$. However, unilateral optic neuritis, short-segment myelitis and other limited forms of neurological syndromes do not exclude NMOSD diagnosis ${ }^{[69]}$. The course is usually relapsing $(90 \%)$, with increasing burden of impairment resulting from incomplete attack recovery ${ }^{[70]}$, and it is influenced in particular by onset age, onset phenotype, and ethnicity ${ }^{[7]}$. In most patients with a diagnosis of NMOSD, AQP4-Abs are detectable in serum, reflecting the autoimmune pathogenesis of the disease ${ }^{[2]}$. However, seronegative cases are also part of the spectrum, and often represent a diagnostic challenge, with unpredictable disease course and final outcome. The development of cell-based assays using transfected cells and a full-length conformationally intact MOG has allowed the identification of serum ${ }^{[7]}$ and, more rarely, $\mathrm{CSF}^{[72]}$ antibodies to MOG in a proportion of patients with NMOSD. However, the clinical spectrum associated with MOGAbs encompasses a broadening range of phenotypes, including NMOSD and partial forms of the disease (prevalent in adults) and ADEM (prevalent in children) ${ }^{[73-76]}$.

In cases positive for MOG-Abs, isolated optic neuritis $(\mathrm{ON})$ is the most common onset presentation (55\%$64 \%$ ), with simultaneous bilateral involvement in $34 \%-42 \%$ of patients ${ }^{[76-78]}$, followed by acute transverse myelitis (22\%-37\%), which typically presents as a LETM with enhancement with blurred margins, the so called "cloud-like enhancement". Simultaneous ON and myelitis $(8 \%)^{[79]}$, an ADEM-like presentation particularly in children, and, more rarely, brainstem presentations ${ }^{[78]}$ and encephalitis ${ }^{[77,80]}$ are other clinical phenotypes associated with MOG-Abs positivity. Disease course can be either monophasic or relapsing (30\%-70\% of cases), with relapses occurring most frequently in the first year after onset and influenced by acute treatment choices ${ }^{[76,78]}$. Relapses are considered less common in this condition than in AQP4Abs-positive NMOSD, manifest more common with ON, and have a great impact on disability ${ }^{[81]}$. Up to now, only monitoring of MOG-Abs titer has been proposed as a possible predictor of disease course. In particular, disappearance of MOG-Abs in serum is considered prognostic of cessation of relapses ${ }^{[77,82]}$, although seropositivity can be maintained over years even without clinical activity ${ }^{[83]}$. On the other hand, MOG-Abs titer at onset does not predict the future disease course in terms of risk of relapses or final outcome $^{[84]}$. As a consequence, antibody titers can help treatment decisions but do not seem reliable enough to be used in the clinical setting for patients' management. MOG-Abs related disorders usually have a favorable prognosis, with a full/good recovery observed in $78 \%$ of cases. However, patients can be left with significant sphincter/erectile dysfunction, cognitive impairment, and poor visual acuity, mainly driven by onset attack. Good recovery is more frequent in cases with unilateral ON or ADEM and in younger patients $^{[78]}$.

For the aforementioned characteristics of NMOSD and related conditions, it is evident that there is a need to improve prediction of disease course and short-/long-term prognosis.

Previous reports described astrocytic damage as a primary pathologic process in NMOSD, which is supported by the presence of AQP4-Abs in the serum of most patients $(68 \%-91 \%)^{[85]}$. These antibodies 
target aquaporin-4, an integral membrane protein of astrocytes and ependymal cells of CNS, and have pathogenic potential ${ }^{[6]}$. As a consequence, soluble GFAP, which reflects astrocytic damage, has been proposed as a useful disease-severity marker in subjects with AQP4-Abs related NMOSD ${ }^{[86]}$. In addition, subjects positive for both AQP4-Abs or MOG-Abs show an increase in CSF myelin basic protein in comparison with MS cases, reflecting the concomitant presence of myelin injury ${ }^{[87]}$. This concept is supported by the demonstration that the main target in MOG-Abs related conditions is located on the surface of myelin sheath and in the plasma membrane of oligodendrocytes ${ }^{[8]}$.

In this scenario, the possible concomitant increase in biomarkers reflecting axonal damage (i.e., NfL) has appeared worthy of investigation in the scientific community. A possible implication of axonal damage in patients with NMOSD was first suggested by Wang et al. ${ }^{[87]}$, who demonstrated an increase in CSF $\mathrm{NfH}$ and NfL in this disorder. However, this study did not explore serum NfL levels and also did not distinguish patients according to antibody status, which might influence tissue damage according to the specific target site. We recently analyzed serum NfL levels in patients with NMOSD and related disorders, and when comparing AQP4-Abs-positive, MOG-Abs-positive and seronegative patients, we observed increased serum NfL levels in patients with AQP4-Abs and MOG-Abs ${ }^{[89]}$. In particular, we detected higher NfL levels in AQP4-Abs-positive subjects, possibly reflecting the prominent axonal damage consequent to astrocytic and cellular injury, and consequently explaining the severe clinical phenotype/evolution usually described in these subjects. On the other hand, we also detected relatively increased levels of NfL in MOG-Abs-positive patients, suggesting the concomitant presence of axonal damage in this disorder and potentially explaining the long-term disability observed in some MOG-Abs-positive cases ${ }^{[8]}$. We then replicated these observations focusing on 38 MOG-Abs-positive patients and assessing serum and CSF $\mathrm{NfL}$ concentration according to clinical/paraclinical characteristics to investigate NfL as a biomarker of disease severity in this condition ${ }^{[90]}$. We confirmed previous observations on the increase in serum NfL levels in patients with MOG-Abs compared with healthy controls, providing more data on the concomitant presence of axonal damage in this disorder. In addition, when analyzing both serum and CSF samples, we observed a significant correlation between NfL levels in paired samples, supporting the analysis of serum as a reliable and more accessible biological fluid. Even more interestingly, we demonstrated that serum NfL values correlated with attack severity and might predict long-term outcome in patients with MOG-Abs ${ }^{[90]}$. These observations support the broader use of NfL as an accessible and repeatable biomarker of tissue damage in MOG-Abs related conditions, where it is essential to improve the prediction of short- and longterm prognosis. More recently, the analysis of NfL in a group of 33 NMOSD patients (30 seropositive for AQP4-Abs) reported increased levels in comparison with those detected in healthy controls together with a significant correlation between serum and CSF values and a significant association between NfL levels and age. In addition, serum NfL levels were increased during relapses and correlated with EDSS score but were not influenced by treatment and did not predict relapse occurrence in the subsequent year after sampling ${ }^{[91]}$. Altogether, these observations expand the utility of $\mathrm{NfL}$ as a possible disease activity biomarker also in NMOSD and related conditions.

\section{CONCLUSION}

NfL recently emerged as a promising biomarker in the spectrum of demyelinating CNS conditions, in particular after the development of high-sensitivity techniques, which allow us to measure and monitor serum levels over time. NfL values allow us to distinguish patients $v s$. healthy controls, as confirmed by a recent meta-analysis examining 10 studies focused on NfL in CSF and 4 studies on NfL in serum ${ }^{[92]}$. In addition, NfL levels show a correlation with clinical and radiological disease activity and help to predict MS conversion in patients with a first demyelinating event. Finally, different studies support their role in predicting future disability/long-term prognosis and in monitoring therapeutic response, further supporting their role in clinical practice. Additional evidence is needed to clarify whether CSF/blood $\mathrm{NfL}$ assessment is a prognostic/predictive tool in MS patients independently from currently available 
biomarkers. Recent data on the presence of axonal damage also in patients with antibodies targeting astrocytes (AQP4-Abs) or oligodendrocytes (MOG-Abs) further extend the possible use of this biomarker in quantifying disease activity in these conditions, although their role in predicting disease course and long-term prognosis in these disorders has yet to be clarified.

\section{DECLARATIONS}

\section{Authors' contributions}

Analysis and interpretation of data and drafting the manuscript: Bozzetti S, Ferrari S, Gajofatto A

Design and conceptualization of the review, analysis and interpretation of data, and drafting the manuscript: Mariotto S

Read and approved the final manuscript: Bozzetti S, Ferrari S, Gajofatto A, Mariotto S

\section{Availability of data and materials}

Not applicable.

\section{Financial support and sponsorship}

None.

\section{Conflicts of interest}

All authors declared that there are no conflicts of interest.

\section{Ethical approval and consent to participate}

Not applicable.

\section{Consent for publication}

Not applicable.

\section{Copyright}

(c) The Author(s) 2021.

\section{REFERENCES}

1. Thompson AJ, Banwell BL, Barkhof F, Carroll WM, Coetzee T, et al. Diagnosis of multiple sclerosis: 2017 revisions of the McDonald criteria. Lancet Neurol 2018;17:162-73.

2. Wingerchuk DM, Banwell B, Bennett JL, Cabre P, Carroll W, et al; International Panel for NMO Diagnosis. International consensus diagnostic criteria for neuromyelitis optica spectrum disorders. Neurology 2015;85:177-89.

3. Krupp LB, Tardieu M, Amato MP, Banwell B, Chitnis T, et al; International Pediatric Multiple Sclerosis Study Group. International Pediatric Multiple Sclerosis Study Group criteria for pediatric multiple sclerosis and immune-mediated central nervous system demyelinating disorders: revisions to the 2007 definitions. Mult Scler 2013;19:1261-7.

4. Petzold A, Plant GT. Diagnosis and classification of autoimmune optic neuropathy. Autoimmun Rev 2014;13:539-45.

5. Transverse Myelitis Consortium Working Group. Proposed diagnostic criteria and nosology of acute transverse myelitis. Neurology 2002;59:499-505.

6. Lennon VA, Kryzer TJ, Pittock SJ, Verkman AS, Hinson SR. IgG marker of optic-spinal multiple sclerosis binds to the aquaporin-4 water channel. J Exp Med 2005;202:473-7.

7. Reindl M, Waters P. Myelin oligodendrocyte glycoprotein antibodies in neurological disease. Nat Rev Neurol 2019;15:89-102.

8. Gaetani L, Blennow K, Calabresi P, Di Filippo M, Parnetti L, et al. Neurofilament light chain as a biomarker in neurological disorders. J Neurol Neurosurg Psychiatry 2019;90:870-8.

9. Disanto G, Barro C, Benkert P, Naegelin Y, Schädelin S, et al; Swiss Multiple Sclerosis Cohort Study Group. Serum neurofilament light: a biomarker of neuronal damage in multiple sclerosis. Ann Neurol 2017;81:857-70.

10. Gaiottino J, Norgren N, Dobson R, Topping J, Nissim A, et al. Increased neurofilament light chain blood levels in neurodegenerative neurological diseases. PLoS One 2013;8:e75091.

11. Mattsson N, Cullen NC, Andreasson U, Zetterberg H, Blennow K. Association between longitudinal plasma neurofilament light and neurodegeneration in patients with alzheimer disease. JAMA Neurol 2019;76:791-9. 
12. Staffaroni AM, Kramer AO, Casey M, Kang H, Rojas JC, et al. Association of blood and cerebrospinal fluid tau level and other biomarkers with survival time in sporadic Creutzfeldt-Jakob disease. JAMA Neurol 2019;76:969-77.

13. Steinacker P, Anderl-Straub S, Diehl-Schmid J, Semler E, Uttner I, et al; FTLDc Study Group. Serum neurofilament light chain in behavioral variant frontotemporal dementia. Neurology 2018;91:e1390-401.

14. Poesen K, Van Damme P. Diagnostic and prognostic performance of neurofilaments in ALS. Front Neurol 2019;9:1167.

15. Marques TM, van Rumund A, Oeckl P, Kuiperij HB, Esselink RAJ, et al. Serum NFL discriminates Parkinson disease from atypical parkinsonisms. Neurology 2019;92:e1479-86.

16. Posti JP, Takala RSK, Lagerstedt L, Dickens AM, Hossain I, et al. Correlation of blood biomarkers and biomarker panels with traumatic findings on computed tomography after traumatic brain injury. J Neurotrauma 2019;36:2178-89.

17. Korley FK, Goldstick J, Mastali M, Van Eyk JE, Barsan W, et al. Serum NfL (Neurofilament Light Chain) levels and incident stroke in adults with diabetes mellitus. Stroke 2019;50:1669-75.

18. Mariotto S, Farinazzo A, Magliozzi R, Alberti D, Monaco S, et al. Serum and cerebrospinal neurofilament light chain levels in patients with acquired peripheral neuropathies. J Peripher Nerv Syst 2018;23:174-7.

19. Mariotto S, Gajofatto A, Zuliani L, Zoccarato M, Gastaldi M, et al. Serum and CSF neurofilament light chain levels in antibody-mediated encephalitis. J Neurol 2019;266:1643-8.

20. Varhaug KN, Barro C, Bjørnevik K, Myhr KM, Torkildsen Ø, et al. Neurofilament light chain predicts disease activity in relapsingremitting MS. Neurol Neuroimmunol Neuroinflamm 2017;5:e422.

21. Ashton NJ, Leuzy A, Lim YM, Troakes C, Hortobágyi T, et al. Increased plasma neurofilament light chain concentration correlates with severity of post-mortem neurofibrillary tangle pathology and neurodegeneration. Acta Neuropathol Commun 2019;7:5.

22. Mariotto S, Sechi E, Ferrari S. Serum neurofilament light chain studies in neurological disorders, hints for interpretation. J Neurol Sci 2020;416:116986.

23. Kinkel RP, Dontchev M, Kollman C, Skaramagas TT, O’Connor PW, et al; Controlled High-Risk Avonex Multiple Sclerosis Prevention Study in Ongoing Neurological Surveillance Investigators. Association between immediate initiation of intramuscular interferon beta-1a at the time of a clinically isolated syndrome and long-term outcomes: a 10-year follow-up of the Controlled High-Risk Avonex Multiple Sclerosis Prevention Study in Ongoing Neurological Surveillance. Arch Neurol 2012;69:183-90.

24. Granberg T, Martola J, Kristoffersen-Wiberg M, Aspelin P, Fredrikson S. Radiologically isolated syndrome--incidental magnetic resonance imaging findings suggestive of multiple sclerosis, a systematic review. Mult Scler 2013;19:271-80.

25. Håkansson I, Tisell A, Cassel P, Blennow K, Zetterberg H, et al. Neurofilament light chain in cerebrospinal fluid and prediction of disease activity in clinically isolated syndrome and relapsing-remitting multiple sclerosis. Eur J Neurol 2017;24:703-12.

26. Tortorella C, Direnzo V, Ruggieri M, Zoccolella S, Mastrapasqua M, et al. Cerebrospinal fluid neurofilament light levels mark grey matter volume in clinically isolated syndrome suggestive of multiple sclerosis. Mult Scler 2018;24:1039-45.

27. Pawlitzki M, Sweeney-Reed CM, Bittner D, Lux A, Vielhaber S, et al. CSF-Progranulin and neurofilament light chain levels in patients with radiologically isolated syndrome-sign of inflammation. Front Neurol 2018;9:1075.

28. Matute-Blanch C, Villar LM, Álvarez-Cermeño JC, Rejdak K, Evdoshenko E, et al. Neurofilament light chain and oligoclonal bands are prognostic biomarkers in radiologically isolated syndrome. Brain 2018;141:1085-93.

29. Disanto G, Adiutori R, Dobson R, Martinelli V, Dalla Costa G, et al; International Clinically Isolated Syndrome Study Group. Serum neurofilament light chain levels are increased in patients with a clinically isolated syndrome. J Neurol Neurosurg Psychiatry 2016;87:126-9.

30. Kuhle J, Nourbakhsh B, Grant D, Morant S, Barro C, et al. Serum neurofilament is associated with progression of brain atrophy and disability in early MS. Neurology 2017;88:826-31.

31. Bjornevik K, Munger KL, Cortese M, Barro C, Healy BC, et al. Serum neurofilament light chain levels in patients with presymptomatic multiple sclerosis. JAMA Neurol 2019;77:58-64.

32. Dalla Costa G, Martinelli V, Sangalli F, Moiola L, Colombo B, et al. Prognostic value of serum neurofilaments in patients with clinically isolated syndromes. Neurology 2019;92:e733-41.

33. Saleem S, Anwar A, Fayyaz M, Anwer F, Anwar F. An overview of therapeutic options in relapsing-remitting multiple sclerosis. Cureus 2019;11:e5246.

34. Lublin FD, Reingold SC, Cohen JA, Cutter GR, Sorensen PS, et al. Defining the clinical course of multiple sclerosis: the 2013 revisions. Neurology 2014;83:278-86.

35. Domingues RB, Fernandes GBP, Leite FBVM, Senne C. Neurofilament light chain in the assessment of patients with multiple sclerosis. Arquivos de Neuro-Psiquiatria 2019;77:436-41.

36. Martínez MA, Olsson B, Bau L, Matas E, Cobo Calvo A, et al. Glial and neuronal markers in cerebrospinal fluid predict progression in multiple sclerosis. Mult Scler 2015;21:550-61.

37. Lycke JN, Karlsson JE, Andersen O, Rosengren LE. Neurofilament protein in cerebrospinal fluid: a potential marker of activity in multiple sclerosis. J Neurol Neurosurg Psychiatry 1998;64:402-4.

38. Kuhle J, Kropshofer H, Haering DA, Kundu U, Meinert R, et al. Blood neurofilament light chain as a biomarker of MS disease activity and treatment response. Neurology 2019;92:e1007-15.

39. Gunnarsson M, Malmeström C, Axelsson M, Sundström P, Dahle C, et al. Axonal damage in relapsing multiple sclerosis is markedly reduced by natalizumab. Ann Neurol 2011;69:83-9.

40. Sormani MP, Haering DA, Kropshofer H, Leppert D, Kundu U, et al. Blood neurofilament light as a potential endpoint in Phase 2 studies in MS. Ann Clin Transl Neurol 2019;6:1081-9. 
41. Khalil M, Teunissen CE, Otto M, Piehl F, Sormani MP, et al. Neurofilaments as biomarkers in neurological disorders. Nat Rev Neurol 2018; 14:577-89.

42. Kuhle J, Disanto G, Lorscheider J, Stites T, Chen Y, et al. Fingolimod and CSF neurofilament light chain levels in relapsing remitting multiple sclerosis. Neurology 2015;84:1639-43.

43. Kuhle J, Plattner K, Bestwick JP, Lindberg RL, Ramagopalan SV, et al. A comparative study of CSF neurofilament light and heavy chain protein in MS. Mult Scler 2013;19:1597-603.

44. Kuhle J, Malmeström C, Axelsson M, Plattner K, Yaldizli Ö, et al. Neurofilament light and heavy subunits compared as therapeutic biomarkers in multiple sclerosis. Acta Neurol Scand 2013;128:e33-6.

45. Kuhle J, Barro C, Disanto G, Mathias A, Soneson C, et al. Serum neurofilament light chain in early relapsing remitting MS is increased and correlates with CSF levels and with MRI measures of disease severity. Mult Scler 2016;22:1550-9.

46. Sejbaek T, Nielsen HH, Penner N, Plavina T, Mendoza JP, et al. Dimethyl fumarate decreases neurofilament light chain in CSF and blood of treatment naïve relapsing MS patients. J Neurol Neurosurg Psychiatry 2019;90:1324-30.

47. Kuhle J, Plavina T, Barro C, Disanto G, Sangurdekar D, et al. Neurofilament light levels are associated with long-term outcomes in multiple sclerosis. Mult Scler 2019;1352458519885613.

48. Håkansson I, Tisell A, Cassel P, Blennow K, Zetterberg H, et al. Neurofilament levels, disease activity and brain volume during follow-up in multiple sclerosis. J Neuroinflammation 2018;15:209.

49. Novakova L, Zetterberg H, Sundström P, Axelsson M, Khademi M, et al. Monitoring disease activity in multiple sclerosis using serum neurofilament light protein. Neurology 2017;89:2230-7.

50. Burman J, Zetterberg H, Fransson M, Loskog AS, Raininko R, et al. Assessing tissue damage in multiple sclerosis: a biomarker approach. Acta Neurol Scand 2014;130:81-9.

51. Barro C, Benkert P, Disanto G, Tsagkas C, Amann M, et al. Serum neurofilament as a predictor of disease worsening and brain and spinal cord atrophy in multiple sclerosis. Brain 2018;141:2382-91.

52. Siller N, Kuhle J, Muthuraman M, Barro C, Uphaus T, et al. Serum neurofilament light chain is a biomarker of acute and chronic neuronal damage in early multiple sclerosis. Mult Scler 2019;25:678-86.

53. de Flon P, Gunnarsson M, Laurell K, Söderström L, Birgander R, et al. Reduced inflammation in relapsing-remitting multiple sclerosis after therapy switch to rituximab. Neurology 2016;87:141-7.

54. Bhan A, Jacobsen C, Myhr KM, Dalen I, Lode K, et al. Neurofilaments and 10-year follow-up in multiple sclerosis. Mult Scler 2018;24:1301-7.

55. Giovannoni G, Turner B, Gnanapavan S, Offiah C, Schmierer K, et al. Is it time to target no evident disease activity (NEDA) in multiple sclerosis? Mult Scler Relat Disord 2015;4:329-33.

56. Giovannoni G, Tomic D, Bright JR, Havrdová E. "No evident disease activity": The use of combined assessments in the management of patients with multiple sclerosis. Mult Scler 2017;23:1179-87.

57. Delcoigne B, Manouchehrinia A, Barro C, Benkert P, Michalak Z, et al. Blood neurofilament light levels segregate treatment effects in multiple sclerosis. Neurology 2020;94:e1201-12.

58. Salzer J, Svenningsson A, Sundström P. Neurofilament light as a prognostic marker in multiple sclerosis. Mult Scler 2010;16:287-92.

59. Norgren N, Sundström P, Svenningsson A, Rosengren L, Stigbrand T, et al. Neurofilament and glial fibrillary acidic protein in multiple sclerosis. Neurology 2004;63:1586-90.

60. Trentini A, Comabella M, Tintoré M, Koel-Simmelink MJ, Killestein J, et al. N-acetylaspartate and neurofilaments as biomarkers of axonal damage in patients with progressive forms of multiple sclerosis. J Neurol 2014;261:2338-43.

61. Damasceno A, Dias-Carneiro RPC, Moraes AS, Boldrini VO, Quintiliano RPS, et al. Clinical and MRI correlates of CSF neurofilament light chain levels in relapsing and progressive MS. Mult Scler Relat Disord 2019;30:149-53.

62. Sellebjerg F, Börnsen L, Ammitzbøll C, Nielsen JE, Vinther-Jensen T, et al. Defining active progressive multiple sclerosis. Mult Scler 2017;23:1727-35.

63. Martin SJ, McGlasson S, Hunt D, Overell J. Cerebrospinal fluid neurofilament light chain in multiple sclerosis and its subtypes: a metaanalysis of case-control studies. J Neurol Neurosurg Psychiatry 2019;90:1059-67.

64. Romme Christensen J, Komori M, von Essen MR, Ratzer R, Börnsen L, et al. CSF inflammatory biomarkers responsive to treatment in progressive multiple sclerosis capture residual inflammation associated with axonal damage. Mult Scler 2019;25:937-46.

65. Ferraro D, Guicciardi C, De Biasi S, Pinti M, Bedin R, et al. Plasma neurofilaments correlate with disability in progressive multiple sclerosis patients. Acta Neurol Scand 2020;141:16-21.

66. Williams T, Zetterberg H, Chataway J. Neurofilaments in progressive multiple sclerosis: a systematic review. J Neurol 2020; doi: 10.1007/ s00415-020-09917-x.

67. Sato DK, Lana-Peixoto MA, Fujihara K, de Seze J. Clinical spectrum and treatment of neuromyelitis optica spectrum disorders: evolution and current status. Brain Pathol 2013;23:647-60.

68. Flanagan EP. Neuromyelitis optica spectrum disorder and other non-multiple sclerosis central nervous system inflammatory diseases. Continuum (Minneap Minn) 2019;25:815-44.

69. Huh SY, Kim SH, Hyun JW, Jeong IH, Park MS, et al. Short segment myelitis as a first manifestation of neuromyelitis optica spectrum disorders. Mult Scler 2017;23:413-9.

70. Wingerchuk DM, Hogancamp WF, O’Brien PC, Weinshenker BG. The clinical course of neuromyelitis optica (Devic's syndrome). Neurology 1999;53:1107-14.

71. Palace J, Lin DY, Zeng D, Majed M, Elsone L, et al. Outcome prediction models in AQP4-IgG positive neuromyelitis optica spectrum 
disorders. Brain 2019;142:1310-23.

72. Mariotto S, Gajofatto A, Batzu L, Delogu R, Sechi G, et al. Relevance of antibodies to myelin oligodendrocyte glycoprotein in CSF of seronegative cases. Neurology 2019;93:e1867-72.

73. Lechner C, Baumann M, Hennes EM, Schanda K, Marquard K, et al. Antibodies to MOG and AQP4 in children with neuromyelitis optica and limited forms of the disease. J Neurol Neurosurg Psychiatry 2016;87:897-905.

74. Sato DK, Callegaro D, Lana-Peixoto MA, Waters PJ, de Haidar Jorge FM, et al. Distinction between MOG antibody-positive and AQP4 antibody-positive NMO spectrum disorders. Neurology 2014;82:474-81.

75. Cobo-Calvo Á, Ruiz A, D’Indy H, Poulat AL, Carneiro M, et al. MOG antibody-related disorders: common features and uncommon presentations. J Neurol 2017;264:1945-55.

76. Mariotto S, Ferrari S, Monaco S, Benedetti MD, Schanda K, et al. Clinical spectrum and IgG subclass analysis of anti-myelin oligodendrocyte glycoprotein antibody-associated syndromes: a multicenter study. J Neurol 2017;264:2420-30.

77. Cobo-Calvo A, Ruiz A, Maillart E, Audoin B, Zephir H, et al; OFSEP and NOMADMUS Study Group. Clinical spectrum and prognostic value of CNS MOG autoimmunity in adults: the MOGADOR study. Neurology 2018;90:e1858-69.

78. Jurynczyk M, Messina S, Woodhall MR, Raza N, Everett R, et al. Clinical presentation and prognosis in MOG-antibody disease: a UK study. Brain 2017;140:3128-38.

79. Cobo-Calvo A, Vukusic S, Marignier R. Clinical spectrum of central nervous system myelin oligodendrocyte glycoprotein autoimmunity in adults. Curr Opin Neurol 2019;32:459-66.

80. Mariotto S, Monaco S, Peschl P, Coledan I, Mazzi R, et al. MOG antibody seropositivity in a patient with encephalitis: beyond the classical syndrome. BMC Neurol 2017;17:190.

81. Juryńczyk M, Jacob A, Fujihara K, Palace J. Myelin oligodendrocyte glycoprotein (MOG) antibody-associated disease: practical considerations. Pract Neurol 2019;19:187-95.

82. López-Chiriboga AS, Majed M, Fryer J, Dubey D, McKeon A, et al. Association of MOG-IgG serostatus with relapse after acute disseminated encephalomyelitis and proposed diagnostic criteria for MOG-IgG-Associated disorders. JAMA Neurol 2018;75:1355-63.

83. de Mol CL, Wong Y, van Pelt ED, Wokke B, Siepman T, et al. The clinical spectrum and incidence of anti-MOG-associated acquired demyelinating syndromes in children and adults. Mult Scler 2019;16:1352458519845112.

84. Cobo-Calvo A, Sepúlveda M, d'Indy H, Armangué T, Ruiz A, et al; REEM Group. Usefulness of MOG-antibody titres at first episode to predict the future clinical course in adults. J Neurol 2019;266:806-15.

85. Wingerchuk DM, Weinshenker BG. Neuromyelitis optica spectrum disorder diagnostic criteria: Sensitivity and specificity are both important. Mult Scler 2017;23:182-4.

86. Takano R, Misu T, Takahashi T, Sato S, Fujihara K, et al. Astrocytic damage is far more severe than demyelination in NMO: a clinical CSF biomarker study. Neurology 2010;75:208-16.

87. Wang H, Wang C, Qiu W, Lu Z, Hu X, et al. Cerebrospinal fluid light and heavy neurofilaments in neuromyelitis optica. Neurochem Int 2013;63:805-8.

88. Brunner C, Lassmann H, Waehneldt TV, Matthieu JM, Linington C. Differential ultrastructural localization of myelin basic protein, myelin/oligodendroglial glycoprotein, and 2',3'-cyclic nucleotide 3'-phosphodiesterase in the CNS of adult rats. J Neurochem 1989;52:296-304.

89. Mariotto S, Farinazzo A, Monaco S, Gajofatto A, Zanusso G, et al. Serum neurofilament light chain in nmosd and related disorders: comparison according to aquaporin-4 and myelin oligodendrocyte glycoprotein antibodies status. Mult Scler J Exp Transl Clin 2017;3:2055217317743098.

90. Mariotto S, Ferrari S, Gastaldi M, Franciotta D, Sechi E, et al. Neurofilament light chain serum levels reflect disease severity in MOG-Ab associated disorders. J Neurol Neurosurg Psychiatry 2019;90:1293-6.

91. Watanabe M, Nakamura Y, Michalak Z, Isobe N, Barro C, et al. Serum GFAP and neurofilament light as biomarkers of disease activity and disability in NMOSD. Neurology 2019;93:e1299-311.

92. Cai L, Huang J. Neurofilament light chain as a biological marker for multiple sclerosis: a meta-analysis study. Neuropsychiatr Dis Treat 2018;14:2241-54 